\title{
La ville comme une (ou deux) langue : de Montréal à Belo Horizonte
}

\section{The City as Language(s): From Montreal to Belo Horizonte}

\author{
JeAn-François Chassay [chassay.jean-francois@uqam.ca] \\ Université du Québec à Montréal, Canada
}

\section{RÉSUMÉ}

En 2012 paraissait au Québec un recueil de nouvelles de Daniel Grenier intitulé Malgré tout on rit à SaintHenri. Le titre réfère à la fois à un vieux quartier ouvrier de Montréal et à une chanson d'un chanteur populaire, Raymond Lévesque, écrite dans les années 1950. Les nouvelles reposant souvent sur l'utilisation d'une langue vernaculaire, on pensera qu'il s'agit d'un ouvrage ayant un ancrage très québécois, et même d'abord montréalais. Pourtant, l'intérêt du livre tient entre autres, et c'est ce que cette communication voudrait montrer, à une réflexion sur la migration et l'altérité qui s'inscrit, de manière large, dans un imaginaire américain. En effet, certaines nouvelles mettent en scène des Brésiliens et leur adaptation, parfois difficile, à Montréal. Mais surtout, la plus longue nouvelle, intitulée "Les mines générales », propose un chassé-croisé entre Montréal et Belo Horizonte. La nouvelle présente le choc culturel - et même ontologique - de Brésiliens à Montréal, mais aussi d'un Montréalais qui les prend en charge et se retrouve avec eux au Brésil. Il s'agira d'examiner les liens interculturels qui traversent le livre, et surtout cette nouvelle, en mettant l'accent sur différents éléments qui en font l'originalité.

\section{MOTS-CLÉS}

Montréal ; ville ; migration ; Brésil ; Belo Horizonte ; Daniel Grenier ; nouvelle

\section{Abstract}

In 2012, Daniel Grenier published in Quebec his first book, a collection of short stories called Malgré tout on rit à Saint-Henri. The title makes reference to an old working class neighbourhood in Montreal and to a Raymond Lévesque song written in the fifties. As the stories are more often than not narrated in a voice close to the vernacular, the collection could be thought of as a particularly «Québécois » book, and even a "Montréalais » one. However, as this communication will try to argue, the specific qualities of the book lay elsewhere and are best shown in the way it grapples with concepts like migration and alterity, which are part of a larger reflexion on a global American imagination. Indeed, in two stories the reader is told about the difficult adaptation of Brazilian immigrants in Montreal. The longest of the two, «Les mines générales », is the tale of an extremely strange round-trip from Montreal to Belo Horizonte, and back. The story is about the cultural - and ontological shock experienced by a Brazilian family arriving in Quebec, but also, in return, about the deep changes suffered by a Montrealer who takes them under his wing and accompanies them back in their country. This communication will investigate the intercultural bonds that appear throughout the book (especially in this story), while putting the emphasis on the different elements that make it an original contribution to Quebec's literature.

\section{KEYWORDS}

Montreal; city; migration; Brazil; Belo Horizonte; Daniel Grenier; short story

REÇU 2015-08-31; ACCEPTE 2016-06-30

Cet article est une version réduite et légèrement réorientée, en fonction du thème de ce volume, d’un texte à paraître dans l'ouvrage suivant : Gilles Dupuis / Klaus-Dieter Ertler: À la carte. Le roman québécois (2010-2015). Frankfurt am Main : Peter Lang, 2016. 
De nombreux recueils qui se publient réunissent des nouvelles dispersées, parfois inédites, souvent déjà parues en revue. Dans pareils cas, la cohérence tient à l'écriture, aux thèmes que l'auteur aborde. Malgré tout on rit à Saint-Henri de Daniel Grenier, dont c'est le premier livre, a été pensé sur un autre modèle ${ }^{1}$. Il s'agit d'un recueil très construit, à la structure soignée, où les jeux de miroir, les échos et les rappels sont nombreux. Je suggère que l'architecture du recueil, dont les nouvelles sont souvent à la limite du fantastique, se pense dans un rapport d'homologie avec la ville. Les textes se déroulent pour l'essentiel à Montréal, en particulier dans le quartier de Saint-Henri. Ancien quartier ouvrier mythique, notamment à cause du portrait qu'en a fait la romancière Gabrielle Roy dans les années 1940 avec son premier roman, Bonheur doccasion ${ }^{2}$. Quartier qui est encore ouvrier, bien qu'il se soit embourgeoisé au cours des deux dernières décennies.

Le recueil reproduit à sa manière la forme fragmentée et labyrinthique de la ville qui exprime un certain désordre par la multiplicité des signes qui s'y déploient, des traces qu'elle produit et des strates sémantiques qui s'y superposent. Néanmoins, elle impose, à travers ces traces et ces signes, des repères, des témoignages, sinon des cicatrices qui font sens. Pour l'individu qui vit la ville au quotidien, la réalité de celle-ci se situe à la fois dans l'espace horizontal de sa structure physique (toujours en transformation) et dans l'espace vertical de son histoire (qui se densifie à mesure que le temps passe).

Une ville s'apprivoise à la manière d'une langue. Une langue qui nous est très étrangère s'entend d'abord comme une suite de sons sans significations. Son apprentissage nous permet peu à peu de découvrir un rythme, puis un sens, à mesure que les mots que nous apprenons s'inscrivent dans une structure cohérente. La connaissance d'une ville repose sur une initiation similaire.

La question de la langue est justement au cour des phénomènes de migration dans deux des nouvelles du recueil, et notamment la plus longue. Je m'arrêterai sur ces deux nouvelles, en particulier sur «Les mines générales » qui fait une quarantaine de pages, ce qui détonne dans un recueil où les autres textes ( 32 au total) oscillent entre 2 et 15 pages.

Au cours des décennies 1980 et 1990, on a souligné avec raison l'importance prise au Québec par la littérature dite " migrante ». Les auteurs constituaient la première ou la deuxième génération d'individus arrivant de l'étranger, déchirés entre deux cultures ou rendant compte parfois du choc subi dans le pays d'accueil - généralement ville d'accueil, puisqu'ils écrivaient presque tous et toutes de Montréal. Malgré les différences, «l'imaginaire migrant se donne essentiellement comme brouillé, écartelé entre des contradictions impossibles à résoudre. [...] En fait, ce sont les catégories mêmes du proche et du lointain, du familier et de l'étranger, du semblable et du différent qui se trouvent confondues. » (Nepveu 1988 : 199-200) Au moment où émergent ces textes, on croirait qu'une nouvelle forme d'imaginaire surgit qui séduit les lecteurs. Pourtant, comme le rappelle Pierre Nepveu, «l'imaginaire québécois lui-même s’est largement défini, depuis les années soixante, sous le signe de l'exil (psychique, fictif), du manque, du pays absent ou inachevé et, du milieu même de cette négativité, s’est constitué en imaginaire migrant, pluriel, souvent cosmopolite» (Nepveu $1988: 200-201$ ).

1 Au moment où ce texte sera publié, il aura aussi fait paraître un roman intitulé L'année la plus longue, aux éditions du Quartanier.

2 Roman célèbre, Bonheur d’occasion a souvent été considéré comme «le premier roman montréalais ». C’est une affirmation indéfendable, mais qui rend bien compte de l'importance qu'il a joué dans l'imaginaire de Montréal. 
L'imaginaire québécois aujourd'hui, en accord avec la réalité sociale, ne pose plus la question du manque de la même façon que dans les années 1960 et 1970. Mais les manifestations d'une crise identitaire, les liens qui unissent l'individu à des phénomènes d'altérité sont monnaie courante. Le recueil de Daniel Grenier a la particularité d'offrir des textes où l'imaginaire migrant est présenté de l'intérieur, par un Québécois francophone.

La nouvelle intitulée "Sur le bout de la langue » s'ouvre sur une interrogation de la narratrice d’origine brésilienne, installée depuis peu à Montréal : comment se fait-il que le taux de suicide soit si élevé dans un pays où rien ne ressemble à l'agressivité endémique qu'elle a connue chez elle? Ainsi, le texte s'ouvre sur le rapport au corps : celui qui s'autodétruit au Québec, celui qui doit vivre avec la violence au Brésil... Le rapport au corps s'exprime aussi sémiotiquement par le choc de la neige et du froid : «La neige, bien installée, ne lui rappelle rien, ce n'est pas une chose à laquelle elle s'identifie, c'est une chose sur laquelle repose cette nouvelle identité qu'elle essaie de construire »(Grenier 2012 : 85). Refaisant en quelques pages son parcours, elle termine sa réflexion en prononçant le mot que le titre de la nouvelle annonçait, " comme une caresse chaude sur la neige de Montréal. Saudade " (Grenier 2012 : 91). Ce mot difficile à traduire en français (exprimant à la fois de la mélancolie et de la nostalgie) devient la contrepartie linguistique de la neige, un élément culturel auquel on ne peut spontanément s'identifier : il existe des choses qui ne font pas partie de nous mais qu'on parvient à apprivoiser au point qu'elles s'intègrent à notre réalité, même si elles seront toujours un peu vécues dans la distance.

Cette courte nouvelle, magnifique, offre un regard neuf sur Montréal de la part d'une étrangère, alors que les immenses « tours d'habitations brunes [de Lîle-des-Sœurs] commencent à ressembler à des centres communautaires colombiens, ou vénézuéliens » (Grenier 2012 : 84). La beauté de Montréal s'en trouve renouvelée grâce à cette femme, fruit de l'immigration : "Elle se dit que c'est peut-être le mot dans le monde qui dit le moins de choses sur ce qu'il veut dire " (Grenier 2012 : 86). C’est cependant la nouvelle «Les mines générales », la plus imposante du recueil, qui inspire de la manière la plus forte le sujet de la migration et de la dualité culturelle.

Le narrateur est un jeune homme, québécois francophone obsédé par la culture brésilienne et par la langue portugaise.

À l'époque, je n'avais jamais visité le Brésil, ni le Portugal d'ailleurs, mais je pouvais tenir tête à n'importe quel Brésilien dans une conversation sur la littérature de son pays et sur les groupes obscurs de punks de la scène de Rio de Janeiro des années quatre-vingt-dix. Je pouvais lui tenir tête jusqu'à ce qu'il se mette à utiliser des subjonctifs futurs et que ma langue s'enfarge sur un verbe estar mal placé. (Grenier 2012 : 106)

On peut avancer l'hypothèse que la langue portugaise, dans sa version brésilienne, constitue le sujet même de ce récit et son embrayeur narratif. Cette importance de la langue et de ses effets sur le texte se note déjà dans le titre qui traduit littéralement le nom de la région du Brésil d'où vient Gustavo et sa famille, Minas Gerais. Le narrateur affirme : " [J]e me rendais compte que la discussion [en portugais] ne m'intéressait pas tant que le fait purement linguistique de la mener à bien. » (Grenier 2012 : 106) Métatextuellement, cette phrase annonce ce qui va suivre : à travers et grâce à la langue portugaise qui porte le récit, il s'agit de conduire cette histoire à bien, l'amener à un dénouement heureux qui verra le narrateur intégrer en lui une autre culture, en plus de la sienne. 
Dans le bus 24 qui traverse la rue Sherbrooke, un homme vient s'asseoir à côté du narrateur qui, par certains signes, se convainc qu'il s'agit d'un Brésilien. Il sort de son sac un livre d'un écrivain du Brésil, en portugais, pour appâter son voisin. Celui-ci réagira effectivement et se déroulera à ce moment une amusante conversation en portugais, mais en partie traduite, fautes de langue incluses, dont voici un extrait :

- Je comprends bien si tu parlasses -

- Hum?

- Je comprends bien quand les gens disent très lentement.

- Qu'est-ce que tu lis?

- Quoi?

- O-que-você-está-lennnnndddooo?

- Ahhhh! Desculpe : tu lis Chico Buarque.

- Não. Estou. Não está. On dit « eu estou lendo ».

- Ah oui. Desculpe! Je lis Chico Buarque. Le dernier roman de lui.

(Grenier 2012: 108)

Cet imbroglio linguistique, plutôt comique, en cache un autre beaucoup plus tragique. Cet homme, arrivé à Montréal avec sa femme et ses deux enfants, apprend sur place que ses diplômes d'ingénieur ne sont pas reconnus, contrairement à ce qu’on lui avait annoncé au Brésil. Aux prises avec l'indifférence et le mépris de la bureaucratie locale, l'inertie du système, le couple occupe des emplois médiocres et peine à vivre. «Ils étaient partis du Brésil pour toutes les bonnes raisons et toutes les mauvaises raisons en même temps, comme tout le monde " (Grenier 2012 : 111). À la misère vécue au Québec s'opposait une double-contrainte insupportable au Brésil : «Ils arrivaient de Belo Horizonte, capitale de Minas Gerais, un gigantesque État dans le sud-est du pays, immensément riche à cause de l'exploitation des mines de métaux précieux et immensément pauvre à cause des mines de métaux précieux »(Grenier 2012 : 110). Or, le père de Gustavo, le Brésilien rencontré par le narrateur, a fait de la prison sous la dictature militaire alors que le père de Carol, son épouse, a fait fortune dans les métaux précieux sous cette même dictature. Un rapport antagoniste invivable. Gustavo et Carol subissent donc l'histoire d'un double espace tourmenté et cruel : des raisons politiques au Brésil rendent la situation invivable; le déracinement et la mécompréhension réciproque entre les Brésiliens et les fonctionnaires rendent la situation aussi invivable au Québec. Comment échapper à ce dilemme? Comment échapper à cette réalité grise que vivent les personnages et que déplore le narrateur? Par la littérature : en plongeant dans le conte. Et même, dans le conte de fées.

Évidemment, envisager ce texte comme un conte ne peut être entendu au sens strict. Traditionnellement, le conte est marqué par une clôture nette qui le coupe de l'environnement social, produit des personnages sans épaisseur psychologique, échappe à l'Histoire et conserve souvent un caractère enfantin - même si des adultes y trouvent leur compte. Rien de cela ne paraît compatible avec «Les mines générales ». Au contraire même, on pourrait suggérer que le réalisme de la nouvelle - la situation trop fréquente, hélas, d'immigrés qui débarquent dans un pays en découvrant qu'il n'a rien de l'Eldorado espéré - lui donne sa valeur psychologique et sa crédibilité. À cela, on peut répondre deux choses : d'abord, l’intérêt du texte tient justement à 
cet étrange croisement entre le réalisme le plus cru et la structure du conte de fées, deux genres a priori éloignés, qui rejouent sur le plan générique le croisement entre les deux cultures qui organisent le texte : montréalaise et québécoise d'un côté, brésilienne de l'autre. Ensuite, ce texte ne prend vraiment son sens que si on le situe dans l'économie générale du recueil, où la magie, l'occulte, le fantastique viennent sans cesse pénétrer des récits qui s’inscrivent dans la réalité montréalaise.

Dans l'autobus, le narrateur s'étonne :

j'ai vu le Stade olympique et j'ai pensé à une ville comme Belo Horizonte. Je n'arrivais pas à faire le lien entre les deux, dans ma tête, entre mon stade et leur ville. L’un n'existait pas par rapport à l'autre, il n'y avait aucun moyen de les mettre ensemble, de les faire se rejoindre, même métaphoriquement. Et pourtant, [...] Gustavo et Carol avaient été « là-bas » et maintenant ils étaient « ici ", à me parler, à moi. Je n'arrivais pas à comprendre comment l’un devenait l'autre, tout en étant conscient que la vie n'est rien d'autre qu'une série de ces liens qui ne « devraient pas » exister, en toute logique, en toute probabilité. (Grenier 2012 : 112)

Deux mondes s'opposent qui paraissent irréconciliables, comme souvent dans le conte merveilleux. Il existe « mon » stade et « leur » ville. Pourtant le couple se trouve bien là, et la vie est un tissu de phénomènes improbables, d'événements qui échappent à la logique comme l'affirme le narrateur. La littérature, faisant surgir les « lignes de tensions » de la réalité, télescope ce qui en apparence n’existe pas, cristallise les événements de l'Histoire dont les personnages apparaissent parfois comme des métonymies.

Pour pallier à l'injustice des événements, le narrateur va prendre le destin de cette famille en mains. Il va sauver «son » Brésil. Il est si rapidement impliqué dans leur histoire que Montréal ne lui apparaît plus comme la même ville. « J’étais sur le coin d'un boulevard gris, avec une famille brésilienne, et je connaissais plus de choses sur leur pays et sur leur vie que sur l'extrême est de l'île [de Montréal]. On était ailleurs» (Grenier 2012 : 115). L'altérité est si forte que son paysage s'efface, lui semble tellement éloigné du Brésil qu’il le transporte géographiquement loin de l'Amérique : «J'ai traversé le boulevard et, me plantant à un arrêt d'autobus aux allures de station de train perdue au milieu de l'Europe de l'Est, j'ai allumé une cigarette [...] » (Grenier 2012 : 116)

Le narrateur propose d'abord à Gustavo un jumelage linguistique: un apprentissage réciproque du français et du portugais. Puis, lorsque le couple rencontre des problèmes avec leur propriétaire, il le convainc de venir habiter chez lui à Saint-Henri, un quartier plus intéressant que le bout de l'île. « [À Saint-Henri] il ne pleuvait jamais. » (Grenier 2012 : 120) L'attachement à cette famille, et à travers elle au Brésil, sera tel qu'à cause d'eux, indirectement, il laissera sa compagne. On ne saurait mieux dire que son amour se trouve ailleurs et qu'il séloigne sentimentalement de Montréal. «Tu files le parfait bonheur lusophone, j’espère » (Grenier 2012 : 125) lui dira-t-elle sarcastiquement lors de leur dernière conversation téléphonique.

À force d'entendre parler de Belo Horizonte, son désir d'y aller s'intensifie. Il imagine la vie dans cette région " qui dan[se] au son d'une samba sortie tout droit d'une vieille planche à dessin de Walt Disney ( Grenier 2012 : 123). L'allusion à l'univers édulcoré de Disney renvoie davantage, caricaturalement, au monde du conte de fées et du merveilleux. Par la même occasion, 
cette allusion signale qu'il ne faut pas être dupe : le monde réel n'aboutit pas souvent à un tel happy end, mais la fiction peut tout se permettre, elle a tous les droits.

Ayant obtenu un versement important de sa bourse d'étudiant, il décide de payer un voyage à Belo Horizonte à toute la famille et de partir avec eux. Situation improbable - ne serait-ce que parce qu'on imagine mal Gustavo accepter un pareil cadeau - mais qui participe du conte et, pourrait-on dire, du parcours initiatique que le narrateur doit traverser pour se trouver luimême. Lorsque la famille déménage dans son appartement de Saint-Henri, il classe les livres de littérature brésilienne de Carol dans sa bibliothèque, regardant avec plaisir les noms d'auteurs, les titres, feuilletant les livres, s'arrêtant sur des phrases. «Il y avait de tout, du Paulo Coelho, que j'ai mis sur une tablette sans faire de commentaire, pour ne pas la vexer » (Grenier 2012 : 123) écrit-il. Mais n'est-ce pas d'une certaine façon le voyage initiatique raconté par ce même Coelho dans L'alchimiste, que le narrateur effectue, pour se trouver lui-même, découvrir sa "légende personnelle »? Comme pour Disney, dont il apparait comme un double sud-américain dans ce contexte, Coelho et sa bluette moraliste forme une sorte de rempart contre lequel la nouvelle se définit : le texte ne doit pas se lire, comme chez ces créateurs, au premier degré, mais comme un jeu avec la langue et la culture, une hypothèse sur le merveilleux que recèle potentiellement notre monde. Plus que les personnages de Disney ou de Coelho, si le narrateur traverse des épreuves initiatiques, c'est pour devenir une sorte de Candide heureux.

À Belo Horizonte on le reçoit comme un héros dans la famille de Carol. À l'aéroport il croit voir son ex-compagne, Marion, puis cette silhouette s'estompe, comme si le passé montréalais s'effaçait lorsqu'il met les pieds au Brésil. Il débarque dans le château du bon (?) roi, le beau-père de Gustavo, dont on se rappellera quand même qu'il a fait fortune sous une dictature militaire ( Je voyais presque les policiers à cheval de la dictature foncer sur le pauvre père de Gustavo », Grenier 2012 : 131). Répétons-le : ne soyons pas dupe du conte de fées. Mais cette maison immense et magnifique n'est que le point de départ d’un séjour de rêve, comme s'il s'agissait de traduire un Brésil idéal. Et cet univers merveilleux, il y entre aussi parce qu’il en parle la langue, il le pénètre de l'intérieur. Pensant à Montréal, il se confie à lui-même : "j’étais quelqu'un làbas mais moi-même ici »(Grenier 2012 : 136). La dissociation de personnalité est pleinement assumée.

Trop beau pour être vrai se dit-on, une catastrophe va se produire avant la fin; pourtant, le bonheur sera sans faille. Le narrateur retourne à Montréal une semaine avant la famille et la veille de son départ, le beau-père de Gustavo lui remet en cachette un chèque (au montant qu’on devine énorme) pour que le narrateur s'occupe de ses protégés. En rentrant, il achète un magnifique appartement, dans Saint-Henri bien sûr, et le prépare pour eux. D’un château l'autre : l'immense maison de Belo Horizonte a maintenant son équivalent, moins somptueux, mais propice à un pays davantage égalitaire. Après avoir tout organisé en quelques jours, il va manger dans un restaurant brésilien de Montréal et la nouvelle se termine sur la phrase suivante : "Je me suis reculé dans ma chaise pour [...] me plonger dans le bonheur d'imaginer l'arrivée imminente de ma famille "(Grenier 2012 : 144). Le possessif doit s'entendre comme une parfaite intégration à la culture brésilienne.

Ainsi se termine le conte qui a besoin d'une clôture nette. Car enfin, on peut supposer que Gustavo sera réticent à partager le cadeau de son beau-père et que rien n'assure une suite si heureuse au conte. Qu'importe : la finale vient clore le voyage initiatique du narrateur qui se 
termine, en boucle, par un retour à ses origines. Non seulement montréalaise, mais ancrée dans le quartier de Saint-Henri. Entretemps le Brésil aura complété une métamorphose. Soixante-dix ans après Bonheur d'occasion, la culture à Saint-Henri a subi une forte hybridation.

Le romancier, le nouvelliste, n'est pas un sociologue ou un anthropologue, ce qui ne l'empêche pas d'utiliser les codes propres aux disciplines savantes puisque la littérature a la possibilité d'utiliser l'ensemble des discours qui forment la semiosis sociale. Cependant, la connaissance du monde que l'auteur propose dans une fiction relève d'un phénomène d'imagination qui appelle un écart, que je définis au sens où l’entend Jean Starobinski :

Insinuée dans la perception elle-même, mêlée aux opérations de la mémoire, ouvrant autour de nous l'horizon du possible, escortant le projet, l'espoir, la crainte, les conjonctures, l'imagination est beaucoup plus qu'une faculté d'évoquer des images qui doubleraient le monde de nos perceptions directes : c'est un pouvoir d'écart grâce auquel nous nous représentons les choses distantes et nous nous distançons des réalités présentes. (Starobinski $1970: 205$ )

Dans cet écart produit par l'imaginaire, Daniel Grenier parvient à faire des pont entre Montréal et Belo Horizonte, révélant à la fois ce qui rapproche et oppose les deux villes, mais aussi deux langues et, à travers elle, permet le rapprochement des cultures.

\section{Références bibliographiques}

Grenier, D. (2012). Malgré tout on rit à Saint-Henri. Montréal : Le Quartanier. Nepveu, P. (1988). Lécologie du réel. Montréal : Boréal, « Papiers collés ». Starobinski, J. (1970). La relation critique. Paris : Gallimard, « Tel». 
